\title{
An Extensive Case of Merkel Cell Carcinoma due to Fright of COVID-19
}

\author{
Maria Danielsen $^{a} \quad$ Trine Høgsberg $^{a} \quad$ Henrik Frank Lorentzen $^{a}$ \\ Ann-Kathrine Rahr German Rossau ${ }^{b}$ \\ ${ }^{a}$ Department of Dermatology, Aarhus University Hospital, Aarhus, Denmark; ${ }^{b}$ Department \\ of Dermatology, Aalborg University Hospital, Aalborg, Denmark
}

\section{Keywords}

Skin cancer $\cdot$ COVID-19 $\cdot$ Merkel cell carcinoma $\cdot$ Case report

\begin{abstract}
Merkel cell carcinoma (MCC) is a subtype of nonmelanoma skin cancer (NMSC) with increasing incidence. Clinically, MCC resembles other far less-aggressive NMSCs, and the pathogenesis is still not understood completely. Rapid diagnosis and treatment are essential to improve overall survival. We present a case report of a 74-year-old female, who had noticed a rapidly growing, oozing tumor on her right flank. She was hesitant to contact the dermatology ward where she had regular checkups as she was afraid of contracting COVID-19. This was in the beginning of the COVID-19 pandemic. At presentation, she had a large exophytic MCC on her right flank and multiple metastases. The disease was at a late stage, and palliative care was the only treatment option left. With this case, we wish to report a rather uncharacteristic location and size of an MCC tumor and suggest that fear of the pandemic and the COVID-19 lockdown has impacted dramatically on attendance of symptomatic patients.
\end{abstract}

\section{Introduction}

MCC is a rare and very aggressive, metastatic prone form of nonmelanoma skin cancer (NMSC) with an estimated incidence at 0.23 per 100,000 people in Caucasian populations, and the incidence of the first primary MCC is slightly higher in men than females in all ethnic groups $[1,2]$. MCC is primarily seen on sun-exposed areas of elderly Caucasians with most cases occurring after the age of 50 and usually presents as a rapidly enlarging, solitary, 
irregular flesh-colored or bluish-red nodule. It is often similar in appearance to other more common NMSCs such as basal cell carcinoma with teleangiectasias and a smooth and shiny surface [2]. Histology confirms the diagnosis, and treatment depends on stage of disease. Herein, we present a case where fear of contracting the COVID-19 virus resulted in delayed diagnosis of fatal disease.

\section{Case Presentation}

A 74-year-old female patient with a history of pilocytic astrocytoma in 2001, nonsmallcell lung carcinoma (T3 N0 M0) in 2010, and vaginal squamous cell carcinoma (T1b N1 M0) in 2013 presented in the dermatologic outpatient clinic with a rapid growing skin tumor (shown in Fig. 1) on the right flank. During childhood, the patient had been treated with arsenic for her psoriasis. She had had multiple NMSCs in her adult life, including both basal cell carcinomas, squamous cell carcinomas, and morbus Bowen. For this reason, she had regular skin checkups at the outpatient clinic.

The patient had first noticed the tumor 5 months prior to the visit in the outpatient clinic but had hesitated to seek help due to the COVID-19 pandemic. At the time of presentation, the tumor was exophytic, measuring $7.5 \times 5 \mathrm{~cm}$ and protruding $3-4 \mathrm{~cm}$ above the skin surface. On clinical examination, multiple smaller lesions suspicious for NMSC and an enlarged lymph node conglomerate in the right axilla were observed. Biopsies from the exophytic tumor were performed, and immunohistochemistry staining showed a positive reaction for pancytokeratin AE1/AE3 and synaptophysin. CK-20 staining was also performed and showed perinuclear dotlike positivity compatible with an MCC. Testing for the Merkel polyoma antigen was not performed.

Diagnostic workup included an (18)F-fluorodeoxyglucose (FDG) positron emission tomography (PET)/computed tomography (CT) scan which showed stage 4 disease with increased FDG activity in multiple lymph nodes, especially in the conglomerate in the right axilla, in bones, in the right mamma, in the liver, and in the exophytic process on the right flank (shown in Fig. 2). A lymph node extirpation was done, and histology showed disseminated neuroendocrine tumor consisting with a primary MCC.

The patient was referred to the plastic surgery department for surgical excision or debulking of the primary tumor due to discomfort and oozing but was found inoperable
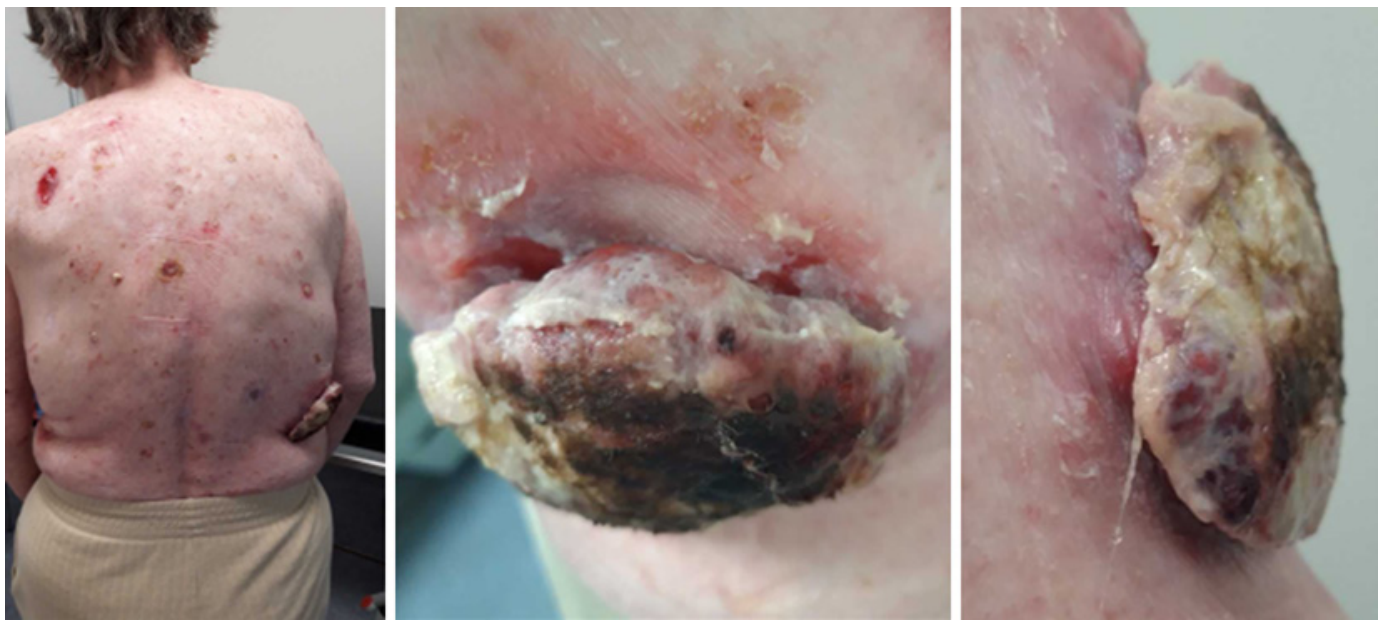

Fig. 1. Photograph of primary tumor localized on the right flank.

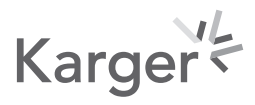


Fig. 2. PET/CT scan with multiple positive foci.

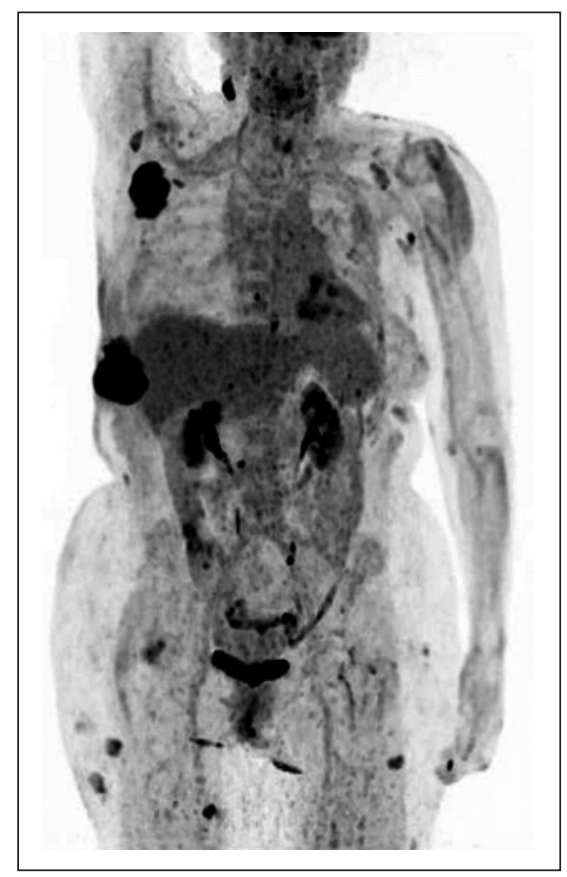

due to disseminated cancer. The patient was then referred to the oncology department for systemic treatment but was deemed ineligible for both chemotherapy and immunotherapy due to bad performance status (PS 3) with rapidly deteriorating health. The patient received palliative radiation therapy (RT) $20 \mathrm{~Gy} / 4$ fractions directed at the primary tumor and the lymph node metastasis in the right axilla. RT decreased the oozing and discomfort, and both the primary and the metastasis shrank significantly in size.

The patient was seen at a follow-up visit in the dermatologic outpatient clinic 6 weeks later. Unfortunately, multiple new rapidly growing skin tumors were observed, including retracting of the right mammary papilla. The patient received another cycle of palliative RT, but at this point, the patient was at a late stage of disease and was declared terminal. The patient succumbed to metastatic MCC a few months later.

\section{Discussion}

The presentation of a MCC measuring $7.5 \times 5 \times 3 \mathrm{~cm}$ at first clinical examination is extremely rare and was partly due to the current global pandemic. Despite the rapid growth of the tumor, the patient thought it was similar to the multiple NMSCs she had previously been treated for and chose not to contact the dermatology ward for a checkup as she was concerned of contracting COVID-19.

The location of the primary tumor on the right flank, in a nonsun-exposed area, is a rather atypical presentation. Previous studies have shown an association with arsenic ingestion/ exposure and development of MCC $[3,4]$. The patient was treated with topical arsenic for psoriasis during childhood which could explain the location of the primary MCC.

The delayed diagnosis resulted in dissemination with distant metastasis hence affecting the possible treatment options. Once MCC is diagnosed, multidisciplinary consultation is common, as seen in this case. Extent of disease at presentation is highly predictive of survival [1]. In localized disease, wide local excision of the primary tumor is the standard of care followed by postoperatively RT. Radiation treatment leads to increased local and regional 
disease control and higher long-term survival rates. Previous studies [2] have shown that survival at 5 years (relative to age- and sex-matched control/population data) was $64 \%$ for local (stage I or II), 39\% for regional nodal (stage III), and 18\% for distant metastatic (stage IV) disease. In particular, extent of nodal involvement has been shown to be a strong prognostic factor, and patients with clinically negative nodal disease should have sentinel lymph node biopsy performed [5]. Any distant metastatic MCC is very serious and has a very poor prognosis. This patient had late-stage disease at initial diagnosis, and only palliative treatment could be offered.

Health care provision has experienced unprecedented strain due to the COVID-19 pandemic. The impact of the pandemic has entailed downgrading of nonemergency clinical services and necessitated rapid re-deployment of capacity and staff toward hospital wards and intensive care units for management of patients with COVID-19 [6]. In this case, delay of diagnosis was mainly due to the concern of contracting COVID-19 which kept the patient from contacting the hospital, but concurrent lockdown of the population has impacted dramatically on attendance of symptomatic patients. For patients with cancer, delay of surgery has the real potential to increase the likelihood of metastatic disease with some patients' tumors progressing from being curable to noncurable [6].

\section{Statement of Ethics}

The patient has signed written informed consent to publish the case and any accompanying images. The study is exempt from ethics committee approval as it is of a purely descriptive nature.

\section{Conflict of Interest Statement}

The authors have no conflicts of interest to declare.

\section{Funding Sources}

There were no funding sources.

\section{Author Contributions}

M.D. contributed to writing - original draft and conceptualization; A.R. contributed to investigation, conceptualization, and writing - review and editing; T.H. contributed to investigation, review, and editing; H.L. contributed to investigation, review, and editing. All authors read and approved the final manuscript.

\section{Data Availability Statement}

All data generated during this study are included in this article. Further enquiries can be directed to the corresponding author. 


\section{References}

1 Poulsen M. Merkel-cell carcinoma of the skin. Lancet Oncol. 2004 Oct;5(10):593-9.

2 Ramahi E, Choi J, Fuller CD, Eng TY. Merkel cell carcinoma. Am J Clin Oncol. 2013 Jun;36(3):299-309.

3 Ho SY, Tsai YC, Lee MC, Guo HR. Merkel cell carcinoma in patients with long-term ingestion of arsenic. J Occup Health. 2005 Mar; 47(2):188-92.

4 Lien HC, Tsai TF, Lee YY, Hsiao CH. Merkel cell carcinoma and chronic arsenicism. J Am Acad Dermatol. 1999 Oct; 41(4):641-3.

5 Becker JC, Stang A, DeCaprio JA, Cerroni L, Lebbé C, Veness M, et al. Merkel cell carcinoma. Nat Rev Dis Primers. 2017 Oct 26;3:17077.

6 Sud A, Jones ME, Broggio J, Loveday C, Torr B, Garrett A, et al. Collateral damage: the impact on outcomes from cancer surgery of the COVID-19 pandemic. Ann OncolPMCID. 2020 Aug;31(8):1065-74. Epub 2020 May 19. 